\title{
Precise Estimation of Postoperative Cup Alignment from Single Standard X-Ray Radiograph with Gonadal Shielding
}

\author{
Guoyan Zheng ${ }^{1}$, Simon Steppacher ${ }^{2}$, Xuan Zhang ${ }^{1}$, and Moritz Tannast ${ }^{2}$ \\ ${ }^{1}$ MEM Research Center, University of Bern, Stauffacherstrasse 78, CH-3014, Bern, \\ Switzerland \\ guoyan.zheng@ieee.org \\ ${ }^{2}$ Department of Orthopaedic Surgery, Inselspital, University of Bern, Switzerland
}

\begin{abstract}
This paper addresses the problem of estimating postoperative cup alignment from single standard X-ray radiograph with gonadal shielding. The widely used procedure of evaluation of cup orientation following total hip arthroplasty using single standard anteroposterior radiograph is known inaccurate, largely due to the wide variability in individual pelvic position relative to X-ray plate. 2D-3D image registration methods have been introduced to estimate the rigid transformation between a preoperative CT volume and postoperative radiograph(s) for an accurate estimation of the postoperative cup alignment relative to an anatomical reference extracted from the CT data. However, these methods require either multiple radiographs or a radiograph-specific calibration, both of which are not avaiable for most retrospective studies. Furthermore, these methods were only evaluated on X-ray radiograph(s) without gonadal shielding. In this paper, we propose to use a hybrid 2D-3D registration scheme combining an iterative landmark-to-ray registration with a 2D-3D intensity-based registration to estimate the rigid transfromation for a precise estimation of cup alignment. Quantitative and qualitative results evaluated on clinical and cadaveric datasets are given which indicate the validity of our approach.
\end{abstract}

Keywords: postoperative cup alignment, radiograph, 2D-3D registration, iterative landmark-to-ray registration, intensity-based registration.

\section{Introduction}

Two-dimensional (2D) anteroposterior (AP) pelvic radiographs, despite their inferior accuracy in comparison to three-dimensional (3D) techniques based on computed tomography [1], are the standard imaging method for the evaluation of cup orientation following total hip arthroplasty (THA) 2 [3], largely due to the simplicity, availability, and minimal expense associated with acquiring these images. While plain pelvic radiographs are easily obtained, their accurate interpretation is complicated by the wide variability in individual pelvic position relative to the X-ray plate [1] (see Fig. 1(a) for a detailed explanation). In THA, increased pelvic tilt results in a significant decreases in apparent prosthetic cup 

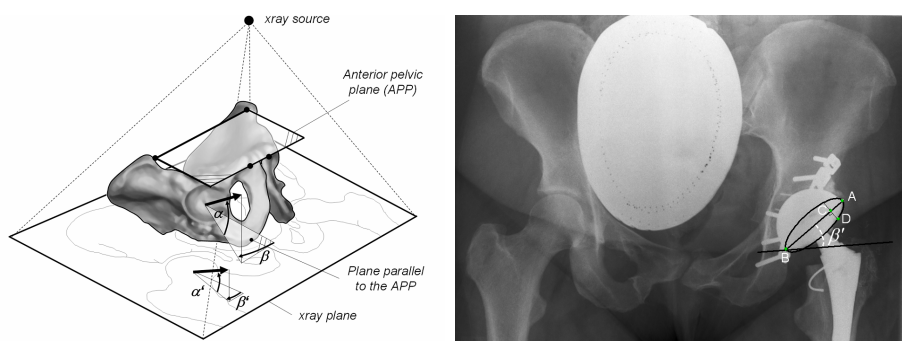

Fig. 1. (a) Two angles ( $\alpha=$ anteversion, $\beta=$ inclination) describe the cup orientation (left image). Due to the large variation of individual pelvic orientation they have to be measured relative to an anatomical reference (the anterior pelvic plane). These standardized values $(\alpha, \beta)$ significantly differ from the cup orientation calculated out of AP pelvic raidograph $\left(\alpha^{\prime}, \beta^{\prime}\right)$; and (b) method from [2] for determining radiographic acetabular cup orientation with measurement lines drawn on a plain X-ray with gonadal shielding (right image). A line is drawn at $1 / 5$ th distance of the maximum diameter $l_{\mathbf{A B}}$ and perpendicular to the major axis $\mathbf{A B}$ of the projected ellipse. It intersects with line $\mathbf{A B}$ at point $\mathbf{C}$ and with the visible arc at point $\mathbf{D}$. And, $\alpha^{\prime}=\arcsin \left(\frac{l_{\mathbf{C D}}}{0.4 l_{\mathbf{A B}}}\right)$.

anteversion and vice versa [4. These position variations affect the accuracy of studies correlating cup position to instability, wear, and osteolysis.

2D-3D image registration methods [5] 6] have been introduced to estimate the rigid transformation between preoperative $\mathrm{CT}$ volume of a patient and postoperative radiograph(s) for an accurate estimation of the postoperative cup alignment relative to an anatomical reference, which is a plane called the anterior pelvic plane (APP) defined by the anterior superior iliac spines (ASIS) and pubic tubercles. The transformation chain in such a method can be summarized by Eq. 1 The estimations of $C u p_{X-\text { ray }}^{\text {Oriention }}$ and $T_{A P P}^{C T}$ are trivial and the challenge lies in the estimation of $T_{C T}^{X-r a y}$, particularly when single standard radiograph with gonadal shielding is used.

$$
C u p_{A P P}^{\text {Orientation }}=T_{A P P}^{C T} \cdot T_{C T}^{X-\text { ray }} \cdot C u p_{X-\text { ray }}^{\text {Orientation }}
$$

In this work, we use the method introduced in [2] to find Cup Orientation and the method published in [7] to find $T_{A P P}^{C T}$. We then propose to use a hybrid 2D-3D registration scheme combining an iterative landmark-to-ray registration 8 with a 2D-3D intensity-based registration 9 to find the rigid transfromation $T_{C T}^{X-\text { ray }}$ between a preoperative CT volume and single standard X-ray radiograph. Our method does not require a radiograph-specific calibration and can work with Xray radiograph with gonadal shielding. The only information that we assume to know about the radiograph is the image scale (pixel $/ \mathrm{mm}$ ) and the distance from the focal point to the imaging plane or to the film. As long as the radiograph is acquired in a standardized way, which is performed in a clinical routine [3], they can be estimated by performing one-time calibration [10]. 


\section{The Proposed Approach}

\subsection{Cup Alignment Estimation Protocol}

Our cup alignment estimaiton protocol includes following five steps:

1. CT data processing and landmark extraction. In this step, we first extract the surface models of both pelvis and femur from the CT volume data and then define region of interest, which will be used in step 4 to exclude the contribution of most of the femur part to the digitally reconstructed radiograph (DRR) - a projection image obtained from the CT volume data by simulating X-ray projection. We then interactively extract two sets of landmarks from the CT data using a custom-made planning tool [13: (a) landmarks for measuring cup orientation, including left and right ASIS, and pubic tubercles; they are used to define the APP; and (b) landmarks for registration, including left and right acetabular centers (by interactive sphere fitting), the pubic symphysis, and the middle of the sacrococcygeal joint (see Fig. 2(a) for details).

2. X-ray radiograph landmark extraction. In this step, two sets of landmarks are interactively picked from the radiograph (see Fig. 2(b) for details): (a) three landmarks for measuring cup orientation as described in 2, which are used to calculate the radiographic cup orientation (see Fig. 1(b)] right); and (b) the corresponding projections of those $\mathrm{CT}$ landmarks for registration, including left and right acetabular centers, the upper border of the symphysis, and the middle of the sacrococcygeal joint. The local coordinate reference and the cone-beam projection model of the radiograph is then established as follows (see Fig. 2(c) for details). The intersection between the line connecting the middle of the sacrococcygeal joint and the upper border of the symphysis and the line connecting the acetabular centers is assumed to be the cone-beam projection center and is taken as the coordinate origin. The central projection line is perpendicular to the radiograph plane and its opposite direction is regarded as Z-axis. With gonadal shielding, it is possible that either the middle of the sacrococcygeal joint or the upper border of the symphysis is occluded. In such a case, a rough estimation is used.

3. Iterative landmark-to-ray registation. Using those landmarks picked from the $\mathrm{CT}$ data and from the radiograph, we perform an iterative landmarkto-ray registation. The estimated rigid transformation is then treated as the starting value for next step.

4. Intensity-based 2D-3D registration. The rigid transformation obtained from last step will be fine-tuned by an intensity-based 2D-3D registration which uses a similarity measure derived from Gibbs random field theory [9.

5. Cup alignment estimation. In this step, we will estimate the cup alignment in relative to the anterior pelvic plane of the patient

\subsection{Iterative Landmark-to-Ray Registration}

Let us denote those landmarks defined in CT volume, i.e., the left and right acetabular centers, the pubic symphysis, and the middle of the sacrococcygeal 


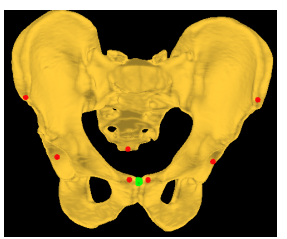

(a)

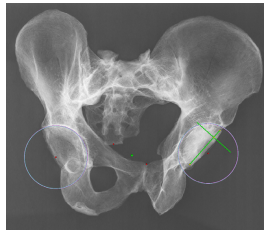

(b)

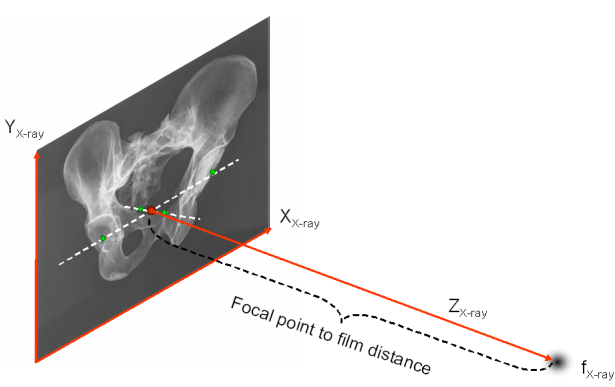

(c)

Fig. 2. (a) Landmarks extracted from CT volume; (b) landmarks extracted from radiograph; and (c) the radiograph coordinate system and the cone-beam projection model

joint, as $v_{C T}^{1}, v_{C T}^{2}, v_{C T}^{3}$, and $v_{C T}^{4}$, respectively; and their corresponding landmarks interactively picked from the radiograph as $v_{X-\text { ray }}^{1}, v_{X-\text { ray }}^{2}, v_{X-\text { ray }}^{3}$, and $v_{X-\text { ray }}^{4}$, respectively. And for each X-ray landmark, we can calculate a projection ray emitting from the focal point to the landmark. We then calculate the length between $v_{C T}^{1}$ and $v_{C T}^{2}$ and denote it as $l_{C T}^{1,2}$, and the shortest distance from $v_{C T}^{3}$ (or $v_{C T}^{4}$, if the upper border of the symphysis on the radiograph is occluded) to line $v_{C T}^{1} v_{C T}^{2}$ and denote it as $l_{C T}^{3,1-2}$ (or $l_{C T}^{4,1-2}$ ). Using the known image scale, we also calculate the length $l_{X-\text { ray }}^{1,2}$ between $v_{X-\text { ray }}^{1}$ and $v_{X-\text { ray }}^{2}$. Then, we do:

Initialization. In this step, we assume that the line connecting the acetabular centers is parallel to the AP pelvic radiograph plane. Using this assumption and the correspondences between the landmarks defined in the CT volume and those picked from the radiograph, we can first compute two points $\bar{v}_{X-\text { ray }}^{1}$ and $\bar{v}_{X-\text { ray }}^{2}$ on the projection rays of $v_{X-\text { ray }}^{1}$ and $v_{X-\text { ray }}^{2}$, respectively, which satisfy:

$$
\bar{v}_{X-\text { ray }}^{1} \bar{v}_{X-\text { ray }}^{2} / / v_{X-\text { ray }}^{1} v_{X-\text { ray }}^{2} ; \text { and }\left|\bar{v}_{X-\text { ray }}^{1}-\bar{v}_{X-\text { ray }}^{2}\right|=l_{C T}^{1,2}
$$

We then find a point $\bar{v}_{X-\text { ray }}^{3}$ on the projection ray of $v_{X-\text { ray }}^{3}$ whose distance to the line $\bar{v}_{X-\text { ray }}^{1} \bar{v}_{X-\text { ray }}^{2}$ is equal to $l_{C T}^{3,1-2}$. A paired-point matching [11] based on $\left\{v_{C T}^{i} ; \mathrm{i}=1,2,3\right\}$ and $\left\{\bar{v}_{X-\text { ray }}^{i} ; \mathrm{i}=1,2,3\right\}$ is performed to calculate the initial rigid transformaton $\bar{T}_{C T}^{X-\text { ray }}$ (see Fig. 3(a) for details). From now on, we assume that all information defined in the radiograph coordinate frame has been transformed into the CT coordinate frame using $\bar{T}_{C T}^{X-\text { ray }}$. We denote the transformed X-ray landmarks as $\left\{\tilde{v}_{X-\text { ray }}^{i}\right\}$ and the transformed X-ray focal point as $\tilde{f}_{X-\text { ray }}$.

Iteration. The following steps are iteratively executed until convergence:

1. For a point $v_{C T}^{i}$, we find a point on the corresponding projection ray of $\tilde{v}_{X-\text { ray }}^{i}$ which has the shortest distance to the point $v_{C T}^{i}$ and denote it as 


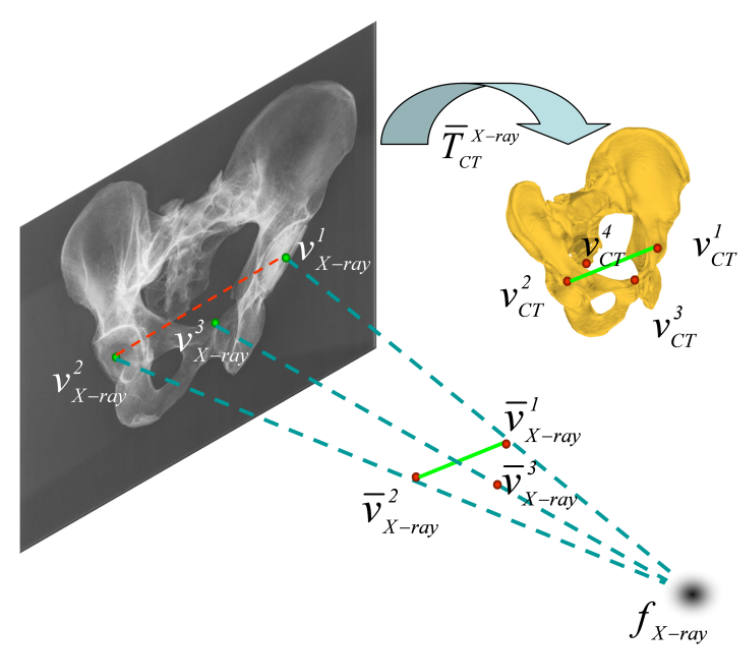

(a)

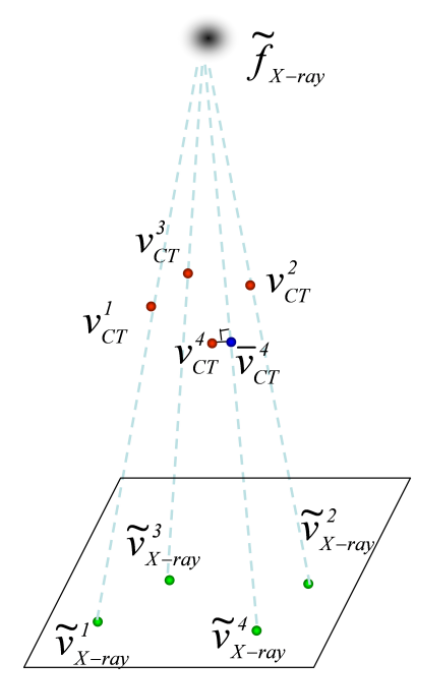

(b)

Fig. 3. Iterative landmark-to-ray registration. (a) schematic view of initialization; and (b) schematic view of finding $3 \mathrm{D}$ point pairs.

$\bar{v}_{C T}^{i}$ (see Fig. 3(b)). We then perform a paired-point matching [11] using the extracted point pairs to compute a rigid transformation $\Delta \tilde{T}_{C T}^{X-\text { ray }}$.

2. We update the radiograph coordinate frame using $\Delta \tilde{T}_{C T}^{X-\text { ray }}$.

\subsection{Intensity-Based 2D-3D Registration}

Without the use of fiducial markers, the iterative landmark-to-ray registration can not fullfill the accuracy requirements of our application and is complemented by an intensity-based 2D-3D registration. The challenge here is the big area occlusion caused by gonadal shielding which creates large differences between the $\mathrm{X}$-ray radiograph and the DRR obtained from the CT volume data by simulating $\mathrm{X}$-ray projection given the current estimation of the rigid transformation, and contains very little useful information to aid registration.

Let us denote $L=\{(i, j): 1 \leqslant i \leqslant I, 1 \leqslant j \leqslant J\}$, an $I \times J$ integer lattice, as the pixel sites of $\mathrm{X}$-ray radiograph and the image value at pixel site $(i, j)$ of the X-ray radiograph as $I_{X-r a y}(i, j)$. Similarly, we denote the image value of the DRR at pixel site $(i, j)$ as $I_{D R R}(i, j)$. Our 2D-3D registration scheme is based on a recently introduced spline-based multi-resolution 2D-3D registration scheme [12] but with different similarity measure. We use a similarity measure that is derived from Gibbs random field theory [9]. It allows us to effectively incorporate spatial inforation and has following form:

$$
S=\sum_{i, j}^{I, J} d_{i, j}^{2}+\sum_{i, j}^{I, J} \frac{1}{\operatorname{card}\left(N_{i, j}^{r}\right)} \sum_{\left(i^{\prime}, j^{\prime}\right) \in N_{i, j}^{r}}\left(d_{i, j}-d_{i^{\prime}, j^{\prime}}\right)^{2}
$$

where $N_{i, j}^{r}$ is a neighborhood defined by 


$$
N_{i, j}^{r}=\left\{\left(i^{\prime}, j^{\prime}\right)\left|\left(i^{\prime}, j^{\prime}\right) \in L,\left(i^{\prime}, j^{\prime}\right) \neq(i, j),\right|\left(i^{\prime}, j^{\prime}\right)-(i, j) \mid \leqslant r\right\}
$$

and $\mathrm{r}$ is a positive integer that determines the size of the neighborhood system. $d_{i, j}$ is the local normalization based difference image value at pixel site $(i, j)$ and is computed by:

$$
\begin{aligned}
& d_{i, j}=\bar{I}_{X-\text { ray }}(i, j)-\bar{I}_{D R R}(i, j) \\
& \bar{I}_{X-\text { ray }}(i, j)=\frac{I_{X-\text { ray }}(i, j)-m_{X-\text { ray }}\left(R_{i, j}^{r}\right)}{\sigma_{X-\text { ray }}\left(R_{i, j}^{r}\right)} ; \bar{I}_{D R R}(i, j)=\frac{I_{D R R}(i, j)-m_{D R R}\left(R_{i, j}^{r}\right)}{\sigma_{D R R}\left(R_{i, j}^{r}\right)}
\end{aligned}
$$

where $m_{X-\text { ray }}\left(R_{i, j}^{r}\right), \sigma_{X-\text { ray }}\left(R_{i, j}^{r}\right)$ and $m_{D R R}\left(R_{i, j}^{r}\right), \sigma_{D R R}\left(R_{i, j}^{r}\right)$ are the mean value and the standard deviation calculated from the intensity values of all sites in the local region $R_{i, j}^{r}$ of the X-ray radiograph and of the associated DRR, respectively. $R_{i, j}^{r}$ has the same size as $N_{i, j}^{r}$ and is defined by:

$$
R_{i, j}^{r}=\left\{\left(i^{\prime}, j^{\prime}\right)\left|\left(i^{\prime}, j^{\prime}\right) \in L,\right|\left(i^{\prime}, j^{\prime}\right)-(i, j) \mid \leqslant r\right\}
$$

To accelerate the registration process, we use the cubic-splines data model described in 12 to compute the multi-resolution data pyramids for both $\mathrm{CT}$ images and X-ray images, the DRRs, as well as the gradient and the Hessian of the similarity measure. The registration is then performed from the coarest resolution until the finest one. And to improve the capture range, we use two different sizes of neighborhood systems: $r=15$ and $r=3$. Starting from the rigid transformation obtained by the iterative landmark-to-ray registation, the similarity measure with the bigger neighborhood system is first minimized via a Levenberg-Marquardt non-linear least-squares optimizer. The estimated rigid transformation is then treated as starting value for optimizing the similarity measure with the smaller neighborhood system.

\section{Experimental Results}

We designed and conducted experiments on two clinical datasets and a cadaveric pelvis dataset. As there are no ground truths availabe for the two clinical datasets, we use them to qualitatively evaluate the effectivenss of the iterative landmark-to-ray registration and the accuracy of the hybrid 2D-3D registration scheme. Fig. 4 shows one example. The input X-ray radiograph is shown in Fig. 4(a) Fig. 4(b) shows the end of the iterative landmark-to-ray registration and the beginning of the intensity-based 2D-3D registration. Both the x-ray radiograph and the $\mathrm{CT}$ volume data are downsampled to $1 / 8$ th of the original sizes. The edges extracted from the DRR are superimposed onto the X-ray radiograph. Fig. 4(b) demonstrates the effectivenss of the iterative landmark-to-ray registration. Fig. 4(c) shows the end of the intensity-based 2D-3D registration. An accurate matching between the X-ray radiograph and the DRR was observed.

To quantitatively evaluate the measurement accuracy of the proposed approach, a cadaveric pelvis and an all polyethylene acetabular component (Charles F. Thackray, Leeds, UK) were used. Before the prosthesis was implanted, we 


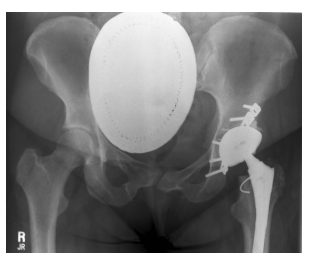

(a)

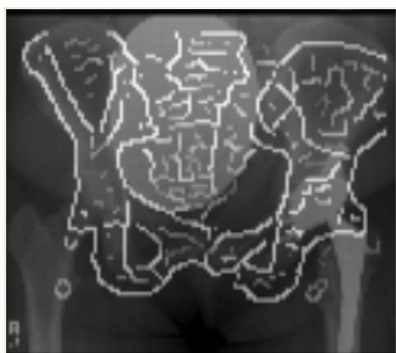

(b)

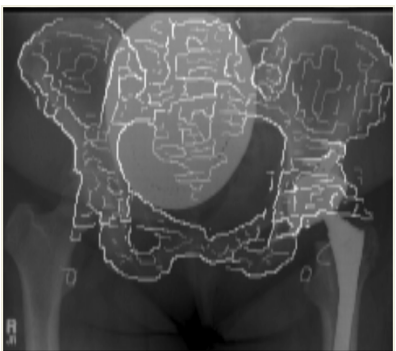

(c)

Fig. 4. (a) X-ray radiograph with gonadal shielding; (b) the beginning of the intensitybased 2D-3D registration. and (c) the end of the intensity-based 2D-3D registration.

Table 1. Experimental results

\begin{tabular}{|c|c|c|c|c|c|c|c|c|c|}
\hline \multicolumn{10}{|c|}{ Difference between radiographic measurements and the ground truths } \\
\hline angle & img_01 & img_02 & img_03 & img_04 & img_05 & img_06 & img_07 & img_08 & img_09 \\
\hline anteversion $\left({ }^{\circ}\right)$ & 13.4 & 1.1 & 2.4 & 20.8 & 10.4 & 19.2 & 17.8 & 12.5 & 9.1 \\
\hline inclination $\left({ }^{\circ}\right)$ & 0.5 & 5.1 & 3.6 & 1.2 & 0.6 & 1.0 & 0.5 & 1.3 & 2.5 \\
\hline \multicolumn{10}{|c|}{ Difference between the estimation results of the first study and the ground truths } \\
\hline anteversion $\left({ }^{\circ}\right)$ & 3.4 & 0.4 & 0.4 & 3.1 & 0.9 & 0.9 & 2.3 & 2.2 & 1.8 \\
\hline inclincation $\left({ }^{\circ}\right)$ & 1.4 & 0.2 & 1.6 & 0.9 & 1.0 & 2.0 & 0.1 & 1.0 & 0.5 \\
\hline \multicolumn{10}{|c|}{ Difference between the estimation results of the second study and the ground truths } \\
\hline anteversion $\left({ }^{\circ}\right)$ & 5.9 & 0.2 & 0.3 & 5.3 & 1.4 & 1.8 & 3.6 & 2.1 & 3.2 \\
\hline inclincation $\left({ }^{\circ}\right)$ & 1.6 & 0.1 & 1.8 & 1.0 & 1.0 & 2.1 & 0.2 & 1.2 & 0.7 \\
\hline
\end{tabular}

did a CT scan of the cadaveric pelvis. After the prosthesis was implanted, we took 9 radiographs by putting the pelvis in different tilt and rotation positions relative to the $\mathrm{X}$-ray plate. To get the ground truth about the prosthesis orientation relative to the anterior pelvis plane of the cadaveric pelvis, we did another CT scan of the pelvis after the prosthesis was implanted. Custom-made software [13. was used to extract the ground truth from the second CT scan. Using these data, we performed two studies. In the first study, each one of the 9 radiograph was used together with the CT scan to estimate the prosthesis orientation and the estimated results were compared to the ground truth. In the second study, to simulate the occlusion caused by gonadal shielding, we intentionally set a region covering $1 / 5$ th-1/3th of the valid image area of each radiograph with constant gray value. We then used each one of these radiographs together with the first CT scan to estimate the prosthesis orientation. The purpose was to see the effect of the gonadal shielding on the accuracy of the proposed approach. The differences between the radiographic measurements and the ground truths, and the differences between the estimated angles in both studies and the ground truths are presented in Table 1. Differences of $11.9^{\circ} \pm 7.0^{\circ}$ were found for the anteversion and differences of $1.8^{\circ} \pm 1.6^{\circ}$ was found for the 
inclination when the radiographic measurements were compared to the ground truths. Our finding is coincident with a recently published finding [1] that radiographic measurement of anteversion is unreliable. With the help of the hybrid 2D$3 \mathrm{D}$ registration scheme, the differences were changed to $1.7^{\circ} \pm 1.1^{\circ}$ for anteversion and $1.0^{\circ} \pm 0.6^{\circ}$ for inclincation, which proved the accuracy of the proposed approach. With the simulated gonadal shielding, the differences were slightly higher but still in the acceptable ranges [1]: $2.6^{\circ} \pm 2.0^{\circ}$ for anteversion and $1.1^{\circ} \pm 0.7^{\circ}$ for inclincation.

\section{Conclusions}

In this paper, we proposed a hybrid 2D-3D registration scheme for accurately estimating postoperative cup alignment using a preoperative CT volume and a standard AP pelvic radiograph. Our method is more appropriate for longterm retrospective study than those have been previously reported [5] 6], which require either multiple radiographs [5] or a radiograph-specific calibration [6], both of which are not available for most retrospective studies. Furthermore, those methods were only evaluated on X-ray radiograph(s) without gonadal shielding, which may post a challenge for them.

\section{References}

1. Kalteis, T., et al.: Position of the acetabular cup-accuracy of radiographic calculation compared to CT-based measurement. Eur. J. Radiol. 58, 294-300 (2006)

2. Pradhan, R.: Planar anteversion of the acetabular cup as determined from plain anteroposterior radiographs. J. Bone Joint Surg. Br. 81-B, 431-435 (1999)

3. Della Valle, C.J., et al.: Primary total hip arthroplasty with a flanged, cemented all-polyethylene acetabular component: evaluation at a minimum of 20 years. J. Arthroplasty 19, 23-26 (2004)

4. Sellers, R.G., et al.: The effect of pelvic rotation on alpha and theta angles in total hip arthroplasty. Contemp. Orthop. 17, 67-70 (1988)

5. LaRose, D., et al.: Post-operative measurment of acetabular cup position using X-ray/CT registration. In: Delp, S.L., DiGoia, A.M., Jaramaz, B. (eds.) MICCAI 2000. LNCS, vol. 1935, pp. 1104-1113. Springer, Heidelberg (2000)

6. Jaramaz, B., Eckman, K.: 2D/3D registration for measurement of implant alignment after total hip replacement. In: Larsen, R., Nielsen, M., Sporring, J. (eds.) MICCAI 2006. LNCS, vol. 4191, pp. 653-661. Springer, Heidelberg (2006)

7. DiGioia, A., et al.: Image guided navigation system to measure intraopertively acetabular implant alignment. Clin. Orthop. Rel. Res. 355, 8-22 (1998)

8. Wunsch, P., Hirzinger, G.: Registration of CAD-models to image by iterative inverse perspective matching. In: ICPR 1996, vol. 1, pp. 78-83 (1996)

9. Zheng, G., et al.: Point similarity measures based on MRF modeling of difference images for spline-based 2D-3D rigid registration of X-ray fluoroscopy to CT images. In: Pluim, J.P.W., Likar, B., Gerritsen, F.A. (eds.) WBIR 2006. LNCS, vol. 4057, pp. 186-194. Springer, Heidelberg (2006)

10. The, B.: Digital radiographic preoperative planning and postoperative monitoring of total hip replacements - techniques, validation and implementation. Doctoral dissertations, University Medical Center Groningen, the Netherlands (2006) 
11. Veldpaus, F.E., et al.: A least-square algorithm for the equiform transformation from spatial marker coordinates. J. Biomech. 21, 45-54 (1988)

12. Jonić, S., et al.: An optimized spline-based registation of a 3D CT to a set of C-arm images. Int. J. Biomed. Imaging , 1-12 (2006) (Article ID 47197)

13. Zheng, G., et al.: A hybrid CT-free navigation system for total hip arthroplasty. Computer Aided Surgery 7, 129-145 (2002) 